\title{
Fallaciously elevated glucose level by handheld glucometer in a patient with chronic kidney disease and hypoglycemic encephalopathy
}

\author{
Praveen Pratap Jadhav, Meera Praveen Jadhav
}

\begin{abstract}
Introduction: The handheld glucometers are commonly used for glucose estimation in acutely ill patients, especially in those with neurological deficit. However, in a few conditions like chronic renal failure, it can give fallacious readings. This has serious implications in management of these patients, which can lead to misdiagnosis of the underlying hypoglycemia. Case Report: Here we present a case of a patient with hypoglycemia who had a fallacious normal glucose level when checked by a handheld glucometer (i.e., elevated on glucometer). The underlying pathology giving rise to this fallacy was presence of chronic kidney disease with high uric acid and low hematocrit. Conclusion: Treating physicians should be aware of such possibilities and take suitable steps for appropriate diagnosis of potentially lifethreatening hypoglycemia.
\end{abstract}

Keywords: Hypoglycemia, Glucometer, Chronic kidney disease, Uric acid

$* * * * * * * * *$

Praveen Pratap Jadhav ${ }^{1}$, Meera Praveen Jadhav²

Affiliations: ${ }^{1} \mathrm{MD}$, Consulting Physician-Omkar Heart Insitute, Nasik, MH, India; ${ }^{2} \mathrm{MS}$, Chief Medical Officer, SurgeonOmkar Heart Institute, Nasik, MH, India.

Corresponding Author: Praveen Pratap Jadhav, Omkar Heart Institute, Gaikwad Mala, Nasik Road, Nasik, Maharashtra, India - 422101; Ph: 91-9822055612; Fax: 91-2532458585; Email: drpraveenj@sify.com

Received: 05 January 2013

Accepted: 27 April 2013

Published: 01 September 2013
Jadhav PP, Jadhav MP. Fallaciously elevated glucose level by handheld glucometer in a patient with chronic kidney disease and hypoglycemic encephalopathy. International Journal of Case Reports and Images 2013;4(9):485-488.

$* * * * * * * * *$

doi:10.5348/ijcri-2013-09-362-CR-6

\section{INTRODUCTION}

Precise diagnosis of acute critically ill unconscious or mentally altered patient is very difficult. Care givers rely on history, examination, imaging and laboratory data for exact diagnosis. However, sometimes laboratory data could be misleading and can lead to hazardous consequences. One such condition, where laboratory results can mislead, is the diagnosis of hypoglycemia in patients with chronic renal failure. Here we present a case where widely used handheld glucometers provided a falsely high capillary blood sugar reading causing diagnostic problems.

\section{CASE REPORT}

A 54-year-old lady was presented with a short history, beginning with altered sensorium and drowsiness and leading to unconsciousness in one hour. She was a diabetic with history of chronic kidney disease on regular treatment. During the initial stages of symptoms, the relatives tried to feed her with oral glucose powder, presuming this to be a hypoglycemic attack. However, the patient was unable to ingest it. The blood sugar level checked on Accu-Chek glucometer (Roche Diagnostics) was $187 \mathrm{mg} / \mathrm{dL}$. Since she did not respond to treatment 
in a few minutes, she was shifted to the hospital for further treatment. She was brought to the hospital deeply unconscious. Her vital parameters were normal, heart rate 100 per minute, blood pressure $110 / 70 \mathrm{mmHg}$ and respiratory rate 18 per minute. She was not responding to deep pain, had flaccid paralysis of all four limbs, pupils were constricted but reacting to light, deep tendon reflexes were depressed and plantar reflexes could not be elicited. Respiratory, cardiac and alimentary system examinations were normal. Her capillary sugar checked on a different Accu-Chek glucometer (Roche Diagnostics) was $167 \mathrm{mg} / \mathrm{dL}$. Differential diagnoses of brain stem cerebrovascular accident, metabolic encephalopathy and uremic encephalopathy were considered. Her blood sample was sent for investigations and her magnetic resonance imaging (MRI) scan of the brain was done. The MRI scan revealed bilateral symmetrical hyper intense lesions in the internal capsule, corona radiata and centrum semiovale, with reduced apparent diffusion coefficient. An hour later her blood reports showed hemoglobin $8.2 \mathrm{~g} / \mathrm{dL}$, normal blood counts, hematocrit $20 \%$, creatinine $6 \mathrm{mg} / \mathrm{dL}$, blood urea nitrogen $96 \mathrm{mg} /$ $\mathrm{dL}$, sodium $132 \mathrm{mEq} / \mathrm{L}$, potassium $5.2 \mathrm{mEq} / \mathrm{L}$, uric acid $9.2 \mathrm{mg} / \mathrm{dL}$, normal hepatic enzymes and ammonia levels and plasma glucose $24 \mathrm{mg} / \mathrm{dL}$. She was infused with $25 \%$ dextrose and then with $500 \mathrm{~mL}$ of $10 \%$ glucose infusion. She responded dramatically in a few minutes. She became conscious, started moving all the four limbs with complete recovery of all neurological deficit. Temporal event of positive neurological improvement after intravenous glucose suggested the diagnosis of hypoglycemic encephalopathy. Patient was treated with initial intravenous and later oral glucose till plasma levels of sugar were stabilized. She was discharged asymptomatic within 24 hours.

\section{DISCUSSION}

Hypoglycemia is a common emergency situation in intensive care units. It is life-threatening, yet easily treatable. The most common wayto diagnosethis condition is with handheld glucometers. Some conditions, however, have the potential to interfere with glucose measurements by handheld glucometers and provide erroneous readings of blood glucose. This can lead to misdiagnosis and wrong treatment in patients with hypoglycemia. Various factors are attributed to altered glucose measurement on the strips. Some common reasons are given in Table 1.

Though most of these factors cause a minor variation in estimated glucose, sometimes, especially with extreme values of these factors, fallacious readings could be obtained and these could be hazardous. In this patient, though it was obvious that patient had hypoglycemia, it was not detected by glucometer testing.

Glucometers use glucose oxidase (GOD) or glucose dehydrogenase (GDH) enzymes to detect the presence of glucose in blood. In these reactions, glucose is metabolized by GOD or GDH to hydrogen peroxide or reduced nicotinamide adenine dinucleotide, respectively. The amount of hydrogen peroxide or rNAD can then be measured by oxidized dye color change or by electrochemical reactions to calculate the amount of glucose present [1]. The glucose oxidase method makes use of one or both of the following reactions:

Table 1: Sources of glucose monitoring inaccuracy

\section{Factors}

Strip factor

Physical factors

Altitude

Temperature

Patient factors

Meter-strip miscoding

Hematocrit

Triglycerides

Uric acid

\section{Mechanism}

Varying storage conditions. Variation of enzyme quantity in different strips.

Increased sensitivity of GOD biosensor strip to oxygen concentration.

Unpredictable response. Brand specific errors

Altered relation between electrical signal produced by strip and reported blood glucose.

Glucose in RBCs is in equilibrium with plasma glucose, but at lower levels. Extreme hematocrit alters the equilibrium.

Takes up volume, thereby reducing the amount of glucose in capillary volume.

Uric acid is oxidized by the electrodes in the tip of the strip.
Pharmacological factors
L dopa, ascorbic acid, icodextrin in dialysate solutions
These drugs interact with the electrodes at the tip of the strip to give high false values.

GOD - glucose oxidase, RBC - red blood cells 


$$
\text { Glucose }+\mathrm{O}_{2} \stackrel{\text { GOD }}{\longrightarrow} \text { gluconic acid }+\mathrm{H}_{2} \mathrm{O}_{2}
$$

$$
\mathrm{H}_{2} \mathrm{O}_{2}+\text { chromogen } \stackrel{\text { peroxidase }}{\longrightarrow} \text { chromophore (color) }+\mathrm{H}_{2} \mathrm{O}
$$

Many conditions can interfere with these chemical reactions happening at the tip of the glucose measuring strip. High levels of uric acid, which is a reducing agent, may result in falsely high glucose readings [1]. Uric acid lowers the value for glucose as determined by the GODPerid method. Bleaching of the final color is the source of such interference in this reaction [2]. Reducing agents other than glucose, such as metabolites of icodextrin, may also be detected by these methods. This is a chemical used in the dialysate for patients with end stage renal failure on long-term peritoneal dialysis, and which is partly absorbed in the circulation. High or low hematocrit values can also lead to falsely abnormal glucose readings. Low hematocrit value has also been shown to increase glucose estimations on handheld glucometer [3]. Both, low hematocrit and high uric acid, are common in critically ill patients as well as in patients with renal failure. Hence, it can be seen that patients with chronic kidney disease may have multiple reasons to get falsely high glucose readings when handheld glucometers are used. This patient, though never dialyzed, had high uric acid and low hematocrit values, which could have led to falsely high glucose levels on the reagent strip. Since uric acid is a reducing agent and though it is implicated as an agent to give falsely elevated glucose in handheld glucometer, we did not come across any reference or case reports describing the same. The MRI scan of the patient was also highly suggestive of hypoglycemic encephalopathy [4].

\section{CONCLUSION}

Hence, we suggest that in patients with chronic kidney disease, especially due to diabetes, effort should be made to diagnose and treat hypoglycemia accurately when they present with altered mental status. This may involve not over-relying on handheld glucometer, using laboratory method to estimate blood glucose for confirmation or empirically treating these patients with intravenous dextrose without laboratory evidence of hypoglycemia.

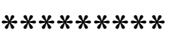

\section{Author Contributions}

Praveen Pratap Jadhav - Substantial contributions to conception and design, Acquisition of data, Analysis and interpretation of data, Drafting the article, Revising it critically for important intellectual content, Final approval of the version to be published

Meera Praveen Jadhav - Substantial contributions to conception and design, Acquisition of data, Analysis and interpretation of data, Revising it critically for important intellectual content, Final approval of the version to be published

\section{Guarantor}

The corresponding author is the guarantor of submission.

\section{Conflict of Interest}

Authors declare no conflict of interest.

\section{Copyright}

(C) Praveen Pratap Jadhav et al. 2013; This article is distributed under the terms of Creative Commons attribution 3.0 License which permits unrestricted use, distribution and reproduction in any means provided the original authors and original publisher are properly credited. (Please see www.ijcasereportsandimages.com/ copyright-policy.php for more information.)

\section{REFERENCES}

1. King DA, Ericson RP, Todd NW. Overestimation by a Hand-Held Glucometer of Blood Glucose Level due to Icodextrin. Isr Med Assoc J 2010 May;12(5):314-5.

2. Chinh NH. Mechanism of interference by uric acid in the glucose oxidase-peroxidase method for serum glucose. Clin Chem 1974 Apr;20(4):499-501.

3. Kang EG, Jeon SJ, Choi SS, Song CJ, Yu IK. Diffusion MR imaging of hypoglycemic encephalopathy. AJNR Am J Neuroradiol 2010 Mar;31(3):559-64.

4. Karon BS, Griesmann L, Scott R, et al. Evaluation of the impact of hematocrit and other interference on the accuracy of hospital-based glucose meters. Diabetes Technol Ther 2008 Apr;10(2):111-20. 
Access PDF of article on other devices other devices

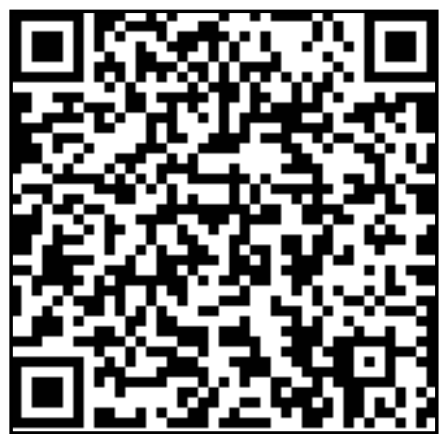

